\title{
Non-destructive evaluation of cement-based materials from pressure-stimulated electrical emission - preliminary results
}

\author{
Kyriazopoulos, A $^{1,2}$, Anastasiadis, C. ${ }^{3}$, Triantis, D. ${ }^{3}$, and Brown, C.J. ${ }^{2 \dagger}$ \\ ${ }^{1}$ Department of Civil Engineering, Technological Educational Institution of Athens, Athens 122 10, \\ Greece \\ ${ }^{2}$ School of Engineering and Design, Brunel University, Uxbridge, UB8 3PH, UK. \\ ${ }^{3}$ Department of Electronics, Technological Educational Institution of Athens, Athens 122 10, \\ Greece \\ ${ }^{\dagger}$ author to receive correspondence
}

Keywords: NDT, laboratory testing. Cement testing, Pressure Stimulated Currents, microcracks. structural health monitoring

\section{Abstract}

This paper introduces the possibility of in-situ assessment of loading and remaining strength in concrete structures by means of measuring discharge of electric current from loaded specimens.

The paper demonstrates that the techniques have been applied to other rock-like materials, but that for the first time they are applied to cement based materials and a theoretical model is proposed in relation to the appearance of electrical signals during sample loading and up to fracture.

A series of laboratory experiments on cement mortar specimens in simple uniaxial compression, and subsequently in bending - hence displaying both tension and compression - are described and show clear correlations between resulting strains and currents measured. Under uniaxial loading there is a well-defined relationship between the pressure-stimulated current (PSC) as a result of a monotonic mechanical loading regime. Similar results are observed in the three-point bending tests where a range of loading regimes is studied, including stepped changes in loading.

While currents can be measured at low strains, best results seem to be obtained when strains approach and exceed yield stress values. 
This technique clearly has immense potential for structural health monitoring of cementbased structures. Both intermittent and continuous monitoring becomes possible, and given an ongoing campaign of monitoring, remaining strength can be estimated.

\section{Introduction}

This paper evaluates the potential use of electrical emission as a method of non-destructive in-situ testing of concrete materials by demonstrating principles applied in laboratorybased tests. In particular, the paper demonstrates that tests on concrete in direct compression, or in compression due to bending show that the concepts are reliable for the assessment of strength and remaining strength of in-situ structures, and suggests that the principles may be developed for use in practical assessment of structures.

Early work on electrical emission from stressed systems was based on efforts to predict earthquake movements. It was readily understood that acoustic, electromagnetic and electrical signals exist which could be detected before and during the fracture of geomaterial; there is some parallel between the electric, electromagnetic and acoustic emissions. Stepanov was the first to observe electric phenomena in the plastic deformation of ionic materials [1], but major experiments involving the study of rocks under stress and the consequent generation of electric currents after cracking were first carried out by Whitworth who demonstrated such an effect in alkali halides [2]. Other researchers $[3,4]$ attributed such phenomena to the piezoelectric effect because their material samples contained piezoelectric components. Electric signals are also observed simultaneously with crack propagation in non-piezoelectric materials indicating the existence of additional electrification phenomena like lattice separation and bond breaking, acceleration of free electrons and other charges [5-7].

The motion of charged dislocations (MCD) [8-11] is one of the basic mechanisms involved in such electrification phenomena but the contribution of these effects cannot be determined individually. The mechanisms interact in a way that is dependent on the material and its mechanical stress history, as well as on its mechanical state. Thus, research readily moved to observations on the state of geomaterial and hence to laboratory tests for transient electrical signals that might predict full-scale events.

Since plastic deformation and micro-fracturing are inextricably linked, the findings of Warwick et al., Ogawa et al., and Enomoto and Hashimoto [12-14] were as expected. 
They measured electric current emissions associated with crack openings and in all cases, microfracture caused discrete electric currents prior to, and during failure. The electrical signals follow the stress variations, and indicate the nature and magnitude of the disturbances in the bulk of the stressed solids. As such, these signals can characterise behaviour. Materials in the transient range between linear and non-linear mechanical behaviour can suffer micro-cracking which leads to irreversible deformations that usually originate from edge dislocations in the bulk of the sample. Dislocations are expected to be electrically neutral, although they may contain various charges of opposite polarities that compensate each other. Such electric charges occur around point defects. For cement-based materials they are capillary pores, air voids and generally the interfacial transition zone which coincides with the regions of high stress concentration in cases of mechanical loadings [2]. The motion of charged dislocations is always present during brittle fracture. Recent laboratory experiments taking recordings of electric signal emissions produced using various modes of uniaxial compressive stress upon rock samples, such as marble and amphibolites, have been analysed [15-20]. The electric signal is detected in the form of a weak electric current using a sensitive electrometer. The current sensor comprises a pair of gold plated electrodes mounted collaterally on the sample under stress. This technique has been characterised as the Pressure Stimulated Currents technique [16], while the weak currents emitted are ascribed as Pressure Stimulated Current (PSC). Similar experimental techniques aiming to bring to light electrical signal emissions during rock failure have also been developed recently. [21,22]

From the very first experimental results it became evident that the characteristics of the electric signals indicate that the procedure of crack formation and development is accompanied by the creation of electric polarization $[15,11]$. On the other hand, the dominant relationship between stress variation rate, the electric charge released and the damage parameter when a sample is subjected to uniaxial compressive stress up to failure, was investigated [16,17]. After systematic measurements, a description of the pressure stimulated electric signals was attempted successfully using statistical mechanics methodology [23] while a linear correlation between the electric charge and the strain before the failure limit was ascertained.

For cement product samples it has also been observed that stress development due to axial compressive load and bending moments induces electric signals as a result of mechanical strain. Wittmann [24] detected a voltage between the compression and the tension zone by 
applying a bending moment on a bar of hardened cement paste. He attributed it to the moisture content of the cement bar.

Preliminary experiments for the study of Pressure Stimulated Currents on Cement-Based Material (CBM) samples like cement mortar and cement paste have been performed [25, 26] but little work exists that relates electric emissions in mortar and cement paste when subjected to loading under low compressive stress [27] or relates ion motion and electrical signal due to the piezoelectric effect [28,29]. The first PSC recordings on CBM samples indicated qualitative similarities with respect to measurements on rock samples [30, 31]. The preliminary results demonstrated that the electric PSC signal on cement samples is evidently stronger. An intense PSC signal with repeated spikes is also detected under high constant uniaxial stress, associated with variations in strain rate [31, 32].

This paper outlines experimental techniques to measure Pressure Stimulated Currents (PSC) on CBM, and compares the results with those obtained from rock materials $[11,16$ 20]. The PSC measurements are systematically recorded while the samples are subjected to temporally varying axial compressive stress, either with a short duration and high repetition rate, or at a low-rate stress increase up to fracture. The experimental results indicate that similarities in the mechanisms of generation and propagation of micro-cracks caused by mechanical stress that have been observed in other materials are also present in cement based materials.

\section{Moving Charged Dislocations (MCD) and Pressure Stimulated Currents (PSC)}

Dislocations and other mechanical imperfections occur in materials when they undergo mechanical action (e.g. tension, compression, bending, and torsion). The electrical neutrality around a physical defect of a deformed material cannot be maintained when the material suffers any further deformation due to mechanical action as the movement in the net of the material structure would generate dipoles of opposite signs and the net polarization would not be zero [10]. Clearly, any net electrical polarization of one sign, must be the result of a net excess of charged dislocations. Since the motion of charge carriers constitutes electric current, it should be expected that detection of electric current emissions is possible when mechanical loading is applied on a sample.

According to the most dominant model that interprets this electrical phenomenon, there is a relationship between electric current and strain rate due to the propagation of arrays of charged edge dislocations through the material during micro-fractures. According 
to this Moving Charged Dislocations (MCD) model, the temporal evolution of the emitted current - PSC $(\mathrm{t})$ - during a process in which a time varying uniaxial load is applied, can be described by a formula of the form:

$$
\operatorname{PSC}(t) \propto \frac{d \varepsilon}{d t}
$$

\section{Equation 1}

where $\frac{d \varepsilon}{d t}$ is the strain rate $[11,33]$. For an elastic material Hooke's law is applicable and for a specimen under uniaxial loading is written in the form:

$$
\sigma(t)=\mathrm{E}_{0} \cdot \varepsilon(t)
$$

Equation 2

where $\mathrm{E}_{0}$ is the modulus of elasticity of the undamaged material. Equation 2 is valid only when the applied stress is lower than the yield stress, $\sigma_{\mathrm{y}}$, i.e. $\sigma \leq \sigma_{\mathrm{y}}$ and for constant $\mathrm{E}_{0}$. For the case that the applied stress is greater than the "yield stress" $\left(\sigma>\sigma_{y}\right)$ the modulus of elasticity gradually decreases and the material enters the damaged range. In this case a variable that quantifies damage is introduced as $\alpha$, the damage variable and is described by the following formula [34]:

$$
\sigma=\sigma_{y}+\mathrm{E}_{0} \cdot(1-\alpha) \cdot\left(\varepsilon-\varepsilon_{y}\right)
$$

Equation 3

where $\varepsilon_{\mathrm{y}}=\sigma_{\mathrm{y}} / \mathrm{E}_{0}$. The damage variable varies between 0 and 1 . When $\alpha=0$, linear elasticity dominates. When $\alpha$ approaches 1 failure occurs. Increasing the value of $\alpha$ in the range 0 to 1 quantifies the weakening of the material and $\mathrm{E}_{0}$ becomes lower and is simply associated with the increase in the number and size of microcracks within the material.

Based on Equations 1 and 2 for the cases that the applied stress increases at a constant rate $\left(\mathrm{d} \sigma / \mathrm{dt}\right.$ and does not exceed the yield stress $\sigma_{\mathrm{y}}$, although PSC are not expected to be emitted, such an abrupt stress change even in this range seems to cause material damage so that PSC emissions can be detected.

For $\sigma>\sigma_{\mathrm{y}}$ the material enters the non-linear region of the stress-strain relation, and permanent changes may take place in the material and the strain rate $\mathrm{d} \varepsilon / \mathrm{dt}$ will not be constant, thus inducing electrification measurable in terms of weak PSC emission. 


\section{Method}

\subsection{Materials}

The material samples were produced at the research laboratory of the Greek cement manufacturer TITAN and included mortars prepared with ordinary Portland cement (OPC). The mixing ratio of the constituents was: 3 parts of fine aggregates (sand with $3 \mathrm{~mm}$ maximum and $0.6 \mathrm{~mm}$ minimum grain size), 1 part of cement and 0.5 parts of water. The fineness of the sand was 2.8 and its specific gravity was 2.6 , while the density was 2.2 . Porosity was evaluated at approximately $8 \%$.

Mixing was performed at a low speed to enable better moisturizing of the cement grains. Quick spinning of the mixture for 1 minute followed, and then the sample was cast into metal moulds with oiled internal surfaces of cubic (for compression tests) and rectangular prismatic (for small beam tests) form. For improved compaction, a desktop vibrator was used in order to remove the air trapped in the bulk of the mixture. The samples remained in the moulds for 24 hours and then they were removed from and placed in a room with a constant ambient temperature $22^{\circ} \mathrm{C}$ and $75-80 \%$ humidity. The samples were stored for 100 days to reach 90-95\% [35] of their total strength.

All samples used for conducting the experimental work were classified depending on their dimensions in the following categories: CM50, CM60, CM70, and CM160. The CM prefix stands for Cement Mortar. The number that follows the prefix describes the length of the sample in $\mathrm{mm}$. (see Table 1 for sample details)

Table 1

\begin{tabular}{|c|c|c|}
\hline Class & Dimensions (mm) & Fracture limit \\
\hline $\mathrm{CM}^{2} 0^{[1]}$ & $50 \times 50 \times 60$ & $35-41 \mathrm{MPa}$ \\
\hline $\mathrm{CM}^{\mathrm{CM}}{ }^{[1]}$ & $50 \times 50 \times 50$ & $25-30 \mathrm{MPa}$ \\
\hline $\mathrm{CM}^{\left[0^{[I]}\right.}$ & $50 \times 50 \times 70$ & around $55 \mathrm{MPa}$ \\
\hline $\mathrm{CM}_{160}{ }^{[2]}$ & $40 \times 40 x 160$ & $(1.4-1.5 \mathrm{kN})$ \\
\hline
\end{tabular}

[1] PSC experiments

[2] Three point bending (BSC) experiments

\subsection{Methods and experimental arrangement}

A range of experiments were performed with different forms of loading, varying both loads and loading rates. Uniaxial monotonic compressive loading was applied in both the linear and nonlinear regions as well as close to sample failure in order to investigate the transition phenomena between the linear and non-linear stages as well as the approaching failure. Two loading regimes were used for uniaxial load:

1. Application of uniaxial compressive loading at a constant stress rate.

In this protocol a stress increase is applied on the sample by loading from the early stress levels up to fracture. The uniaxial stress $\sigma$ is increased linearly at a relatively slow rate described by: 


$$
\sigma=\mathrm{a} \cdot t
$$

Equation 4

where $\mathrm{a}$ is the stress rate, the values of which do not exceed $500 \mathrm{kPa} / \mathrm{s}$; the usual rates are around $100 \mathrm{kPa} / \mathrm{s}$. Failure occurs at time $\mathrm{t}=\mathrm{t}_{\mathrm{f}}=\sigma_{\max } / \mathrm{a}$, where $\sigma_{\max }$ is the ultimate compressive stress strength.

2. Application of uniaxial compressive loading using stepped stress increments.

This test protocol can be applied sequentially from low stress levels up to failure by applying successive stress steps. While the sample is in a state of constant uniaxial stress $\sigma_{\mathrm{k}}$, an abrupt stepwise stress increase of short duration $\Delta \mathrm{t}$ is applied to cause a stress increment $\Delta \sigma=\sigma_{\mathrm{k}+1}-\sigma_{\mathrm{k}}$, where $\sigma_{k+1}$ is the new state after the application of the stress increment. It must be noted that the new stress state $\sigma_{\mathrm{k}+1}$ remains constant until the PSC restores to a low level. The temporal variation of stress $\sigma(\mathrm{t})$, which is recorded during this experimental procedure can be described in a good approximation by the following equation.

$$
\sigma(t)=\left\{\begin{array}{llc}
\sigma_{k}=\text { constant } & \text { for } & t<t_{k} \\
\sigma_{k+1}+b\left(t-t_{k}\right) & \text { for } & t_{k}<t<t_{k+1} \\
\sigma_{k}=\text { constant } & \text { for } & t>t_{k+1}
\end{array}\right\} \quad \text { Equation 5 }
$$

where $\mathrm{b}$ is the stress rate during the stress increase from an initial level $\sigma_{\mathrm{k}}$ to the final value $\sigma_{\mathrm{k}+1}$. Typical values of stress rate $\mathrm{b}$ vary between $1.5 \mathrm{MPa} / \mathrm{s}$ and $5 \mathrm{MPa} / \mathrm{s}$ for the abrupt stress steps. A series of sequential abrupt stress steps can be applied on the sample under test and the PSC emitted can be found for all applied stress ranges levels. However, it is particularly interesting to observe the PSC recordings of stepwise stress application when the stress level is close to the fracture limit. 3. Application of bending loading.

Three-point bending on a CBM beam can be performed in linearly increasing loads up to failure of the beam, or with abruptly increasing loading. The technique adopted here for the experimental loading of the samples is based on the increase of the load in abrupt steps. The beam under test is subjected to loading of constant value (slightly smaller than its ultimate strength). The loading is accomplished by applying a force step increase at a constant rate. It must be noted that the new stress level is close to the strength of the sample and remains constant until the sample fails.

The application of bending has the advantage of simultaneous recording of two electric signals emitted both from the compression and tension zones. Such current emissions are identified as Bending Stimulated Currents (BSC)

\subsection{Measurement}


The experimental arrangement using the PSC technique is depicted in Fig.1. A pair of gold plated copper electrodes is used to collect the signal, and each electrode is placed at the centre of each of two collateral sides of the sample for the current to be detected in a direction perpendicular to the axis on which stress is applied. The electrodes are circular with a $20 \mathrm{~mm}$ diameter. Strain gauges are placed at the centre of each one of the remaining collateral sides of the sample. Teflon plates were added between the stressing system and the sample to provide electrical insulation. Repeated experiments showed that the characteristics of the PSC emitted were identical with or without the Teflon plates.

The experimental arrangement is slightly different when conducting BSC experiments. The electrodes are made again from gold-plated copper of relatively small dimensions compared to the geometry of the samples. They can be placed on the surfaces of both the tension and of the compression zones. The cement mortar beam was supported by two rigid cylindrical rods positioned $70 \mathrm{~mm}$ each from the middle of the beam (see Fig. 2).

In the case of bending two pairs of electrode sets are used for the detection of electric current. As the tensile strength of the mortar is much less than its compressive strength, fracture occurs when a flexural tensile crack is initiated at the bottom of the beam and propagates upwards through the beam $[36,37]$. The Teflon plates are installed between the rods that support the sample and the stressing system, hence there is no impact on the mechanical behaviour of the sample since Teflon does not come into contact with the sample (Figure 2).

The weak currents are measured with the use of sensitive electrometers and the data are stored in the hard disk of a computer via a GPIB channel. The applied bending load is recorded with the use of a Keithley analogue-to-digital data acquisition device (A/D DAQ). The whole setup is placed and protected in a Faraday shield in order to avoid interference from electromagnetic noise.

\section{Results}

\subsection{Laboratory experiments applying uniaxial load}

The low stress rate technique was applied on a number of mortar samples in order to verify whether electric current emissions exist in stressed CBM and if so, whether they have qualitative or quantitative similarities compared to emissions from geomaterials. The experiments were also intended to detect any influence of the stress rate on the PSC.

The Low Stress Rate Technique was applied on cement mortar samples (CM60 class) with dimensions 50mmx50mmx60mm with a fracture limit around $35 \mathrm{MPa}$. The applied stress rate was approximately $102 \mathrm{kPa} / \mathrm{s}$. The sampling rate for the measurements was chosen to be between $2 \mathrm{~s}^{-1}$ and $1 \mathrm{~s}^{-1}$ depending on the experiment. Fig. 3 shows the stress and associated strain with respect to time. It can be observed that after 200 s corresponding to approximately $28 \mathrm{MPa}$ i.e. $80 \%$ of the 
ultimate stress, the strain curve declines from linearity with a strong tendency of the strain rate to increase, thus giving rise to significant deformation in the sample. This means that for $\sigma>\sigma_{\mathrm{y}} \approx 28$ MPa signals the initiation of micro-cracks and damage in the material. The above become clearer in Fig.4 where stress - strain measurements on the same sample are depicted. The Young's modulus for the group of samples used was calculated to be approximately $21.6 \mathrm{GPa}$.

Figure 5 shows the form of the emitted PSC (darker line) and associated strain with respect to time for the same sample. At low stress values (and hence low strain values) corresponding to $\sigma<\sigma_{y}$, the PSC values are very small. When stress-strain becomes non-linear the PSC increases very quickly reaching a peak. Thus, it can be observed that the onset of PSC emission is very strongly related to the onset of significant stiffness changes in the sample. This means that micro-cracks are produced in the material and dislocations begin to move defining the direction of cracking and simultaneously causing charge separations which irreversibly lead to polarizations and hence to current flow. Finally the sample is driven to the ultimate fracture.

Figure 6 depicts the variation of PSC on a logarithmic scale after noise elimination with respect to the relative compressive stress $\hat{\sigma}$ where $\hat{\sigma}=\sigma / \sigma_{\max }$. There are three discrete regions in the PSC curve:

a) The first region for $0.5<\hat{\sigma}<$ (approximately) 0.7 the PSC values show a small exponential current increase of the type $I=I_{0} \exp (\alpha \cdot \hat{\sigma})$ where $I_{0}$ is an electric current constant and $\alpha$ is a characteristic exponent describing the magnitude of the PSC increase. In this region the value of $\alpha$ is approximately equal to 2.5 .

b) The second region for $0.7<\hat{\sigma}<0.9$ there is an intense exponential current increase and the value of $\alpha$ is approximately equal to 27 .

c) The third region is defined for $\hat{\sigma}>0.9$. In this case the PSC increase rate is reducing and a PSC peak appears slightly before fracture.

From the form of the PSC curve, the three basic regions describing the mechanical behaviour of the material can be recognized.

The experiment that follows was designed to detect the influence of the stress rate on the PSC. Two identical C50 class samples with dimensions 50mmx50mmx50mm were used. The former (PSC1) was subjected to uniaxial compressive stress at a constant rate $0.72 \mathrm{MPa} / \mathrm{s}$ and fracture occurred when the stress reached $30 \mathrm{MPa}$. The latter (PSC2) was subjected to compressive stress at a constant rate $0.14 \mathrm{MPa} / \mathrm{s}$ and fracture occurred when the stress reached $25 \mathrm{MPa}$.

Figure 7 depicts two recordings of PSC on a logarithmic PSC axis with respect to time (PSC1 and PSC2), that correspond to the two samples. In the same figure, the temporal variation of the uniaxial compressive stress for two rates, a high stress rate (HSR) and a low stress rate (LSR) is also depicted. The corresponding values of the parameter a (Eq. 4) are respectively $0.72 \mathrm{MPa} / \mathrm{s}$ and 
$0.14 \mathrm{MPa} / \mathrm{s}$. PSC1 starts to be excited significantly from $\mathrm{t}=25 \mathrm{~s}$ that corresponds to a compressive stress of approximately $20 \mathrm{MPa}$ while for the PSC 2 the excitation time is $\mathrm{t}=115 \mathrm{~s}$ and the stress is approximately 17.5 MPa. The above two values of the compressive stress correspond to the values of the yield stress $\sigma_{y}$, taking into account that there is a differentiation in the values of $\sigma_{\max }$ (the ultimate compressive stress strength) of the samples under different loading mode up to fracture. The total electric charge released during the process was calculated for both experiments using the relation:

$Q=\int_{0}^{t_{f}} P S C(t) d t$

Equation 6

The principal characteristic of both PSC recordings is that the electric charges QHSR and QLSR released from the two samples are nearly equal, irrespective of the applied stress rate (QHSR $\approx$ QLSR $\approx .4 .3 \mathrm{nC}$ ). This characteristic property has also been observed in relevant experiments that were conducted on marble samples and has been discussed and interpreted elsewhere [18].

3.2 Laboratory experiments using the step stress technique

Abrupt compressive step loading was applied in order:

i) to certify that PSC emissions might be expected irrespective of the way that loading exerts stress upon a CBM sample and

ii) to study the probable differences in the behaviour of the sample and to determine their causes.

Abrupt stress application causes a high strain rate and consequently might cause the appearance of the PSC peak given that $\mathrm{d} \varepsilon / \mathrm{dt}$ practically diminishes as the stress remains constant.

Three modes of loading were applied on cement mortar samples. A sample of the CM70 class with dimensions $50 \mathrm{mmx} 50 \mathrm{mmx} 70 \mathrm{~mm}$ having a fracture limit around $55 \mathrm{MPa}$ was subjected to the application of abrupt loading steps in four increasing levels up to fracture, and the PSC emissions were recorded. Systematic stress - strain measurements were performed for the determination of yield stress which was found to be $\sigma_{\mathrm{y}}=42 \mathrm{MPa}$. The fourth step led to a final stress of $37 \mathrm{MPa}$, slightly smaller than $\sigma_{\mathrm{y}}$.

The four sequential abrupt stress increments had a constant rate ( $b=5 \mathrm{MPa} / \mathrm{s})$ and care was taken to ensure that the stress rate during each stress increase was the same in order for the PSC peak maxima to be comparable (see Figure 8a). After numerous systematic experiments following this procedure and using the above class of samples the mean final stress level was found that to be 37.5 $\mathrm{MPa}$. This value was proved to be the yield stress $\sigma_{\mathrm{y}}$ corresponding to the CM70 class of samples. When each abrupt compressive loading is applied on the sample, simultaneous and similar abrupt current spikes appear, having peaks at value $\operatorname{PSC}_{\text {peak }}$ (see Figure $8 \mathrm{~b}$ ). It should be noted that the appearance of these small PSC is due to abrupt stressing even at low levels. During microcrack 
creation and the consequent charge production a time-varying microcurrent appears around the microcrack. The recorded PSC is the superposition of such microcurrents whose magnitude reaches a maximum when the concentration of microcracks also reaches a maximum. In particular, when a crack meets a hard aggregate particle such as a sand grain (which is certain after the initial steps), crack branching may occur so that instead of a single crack advancing, a whole family of cracks might gradually be formed. This requires larger forces and absorbs more energy.

Experimental results using the above technique on rock samples as well as on cement-based products, show that after the application of an abrupt stress step a PSC spike is always recorded. When the stress is stabilized at level $\sigma_{\mathrm{k}}$, the PSC gradually decreases. From these results it may be observed that during the first period of time after relaxation of stress at the level $\sigma_{\mathrm{k}}$, the decay of PSC approximation well to an exponential decrease law

$$
\operatorname{PSC}(\mathrm{t})=\mathrm{A} \cdot \exp (-\mathrm{t} / \tau) \quad \text { Equation } 7
$$

where $\tau$ is a relaxation constant and pre-exponential factor $\mathrm{A}$. This relaxation constant $\tau$ is one of the fundamental PSC macroscopic parameters and its value depends on the stress level $\sigma_{\mathrm{k}}$. From the first preliminary experiments presented here the dependence of the PSC relaxation times on the final stress levels has become evident. Indeed, in the 4-step experiment the four relaxation constants had the values $5 \mathrm{~s}, 7 \mathrm{~s}, 9 \mathrm{~s}$ and $13 \mathrm{~s}$ corresponding to stress values $16 \mathrm{MPa}, 23 \mathrm{MPa}, 31 \mathrm{MPa}$ and 37MPa respectively. These values indicate clearly an increase of $\tau$ with respect to the final stress level after the step. This kind of dependence needs a more thorough study in order to investigate the kind of dependence in the regions $\hat{\sigma}<0.7$ and $0.7<\hat{\sigma}$.

To examine the PSC signal emission when CBM samples are under uniaxial compressive stress of a constant value close to the fracture limit, CBM samples of the CM60 class $(50 \mathrm{~mm} \times 50 \mathrm{~mm} \times 60 \mathrm{~mm})$ with a fracture limit between 35 and $41 \mathrm{MPa}$ were used. The samples were stressed up to a value just below the fracture limit. They were kept under this regime for a relatively long time in order that the PSC be stabilized at a minimum background value.

A small step stress increase followed and the sample was led to a new stress regime higher than the previous one by $1-1.5 \mathrm{MPa}$. The sample remained in this regime for a sustained period. The diagram in Figure 9 shows the applied stress steps with respect to time. Step 1 was from $34.5 \mathrm{MPa}$ to 35.7 MPa and Step 2, which was applied after the sample had remained at 35.7 MPa for approximately 2000 seconds, and which caused its failure. The same diagram depicts PSC with respect to the recorded stress. During Step 1 the PSC increases significantly from $7 \mathrm{pA}$ to $78 \mathrm{pA}$ getting to a maximum (peak1) on completion of the stress increase. After stress stabilization at 35.7 $\mathrm{MPa}$, the PSC starts decreasing at a low rate without returning to its initial value of $7 \mathrm{pA}$. While it seems to have relaxed after 550s at around 20pA, Figure 9 shows a noticeable abrupt and wide progressive PSC increase is observed when reaching approximately 95pA (peak 2). The substantial 
difference between the two peaks is that peak 1 appeared as a result of the stepwise stress increase from $34.5 \mathrm{MPa}$ to $35.7 \mathrm{MPa}$ while step 2 is recorded under a regime of constant stress. The simultaneous recording of strain in this experiment may give a macroscopic interpretation to the phenomenon described.

Figure 10 shows both the temporal variation of PSC and strain, so that it becomes evident that the appearance of peak 2 is associated to an intense change in the strain rate in the time window of peak 2. Logically, the increase in the strain rate indicates a consequent PSC increase. This experimental fact verifies qualitatively Eq.1 of the MCD model. The intense increase of the strain rate when peak 2 appears must be associated with the development of macrocracks within the material during the experiment. A large number of pre-existing microcracks of various sizes merge to create macrocracks and the material suffers from an intensely increasing deformation. Microcrack merging leads to more electric charged motion, and hence to greater PSC values.

Figure 11 shows PSC variation with time, conducted on an identical sample after applying four successive stress steps close to the fracture limit. The three spikes corresponding to the three steps before the fracture of the sample are differentiated with respect to the $\mathrm{PSC}_{\max }$ values due to the differentiation of the stress rate and consequently of the strain rate. The PSC relaxes after stabilization of the stress level to continually higher values. When stress reaches the level of 35.7 MPa and stays there for approximately 1000s, a relatively intense current peak appears. This peak, as well as the previous ones, is associated with an intense increase in the strain rate (Figure 12).

The maximum current values (PSC peaks), recorded in the two last experiments, can be correlated with the corresponding values of the mean strain rates $(\mathrm{d} \varepsilon / \mathrm{dt})$ when the peaks appear. Figure 13 shows that this is linear which strongly supports the experimental verification of the MCD model.

\subsection{Application of Three Point Bending Technique}

The variations of both the Bending Stimulated Currents (BSC) for the two opposite sides of the beam due to the load application and of the applied load with respect to time are shown in Figure 14. The BSC signals appeared when a loading step was applied at an increase rate equal to 0,14 $\mathrm{kN} / \mathrm{s}$ up to a final beam loading of $1.3 \mathrm{kN}$. It must be denoted that the value $1.3 \mathrm{kN}$ is slightly smaller than the force necessary to break the beam. (see Table 1). Fig. 14 depicts the appearance of PSC on both opposite sides of the beam due to loading. When the load attains the final value of $1.3 \mathrm{kN}$ then, the two currents start a relaxation process.

The solid curve corresponds to the pair of electrodes positioned on the upper compression surface of the beam with a strain rate $\mathrm{d} \varepsilon / \mathrm{dt}<0$. The dotted curve corresponds to the pair of electrodes positioned on the lower surface of the beam (tensile region where the strain rate is $d \varepsilon / d t>0$ ). It is 
characteristic that the values are of opposite polarity, and this is in agreement with the MCD model because it is expected (Eq. 1) that the electrical signals are proportional to the strain rate.

The maximum value of the current corresponding to the tensile region $\mathrm{BSC}_{\max }^{\mathrm{T}}$ is greater by more than twice of that in the compression region ${ }^{B S C} C_{\text {max }}^{\mathrm{C}}$. This is because the extensive deformation in the lower part of the beam gives rise to a larger number of microcracks and the accompanying dislocations move apart more readily than those in the compressed upper region [36,38]. Systematic measurements show that between the values $\mathrm{BSC}_{\max }^{\mathrm{T}}$ and $\mathrm{BSC}_{\text {max }}^{\mathrm{C}}$, there is a ratio $\mathrm{BSC}_{\max }^{\mathrm{T}} / \mathrm{BSC}_{\max }^{\mathrm{C}} \approx 3$

Figure 14 also demonstrates that both BSC recordings reach a maximum at the same instant, before the applied force reaches its sustained value, after which both bending stimulated currents enter relaxation.

An additional experiment was conducted by applying two steps of increasing loading to a similar sample. In Figure 15, both BSC and load are depicted with respect to time. On making an attempt to apply a third step, the beam failed while BSC spikes were recorded just before failure. The same pattern as before is observed, and as with uniaxial loading, larger values of BSC are observed when the final loading value is greater. In the BSC recordings the peak of the BSC signal appears with a temporal lead of some seconds ( 3 to $10 \mathrm{~s}$ ) before the load gets to its final constant value. This differentiation in temporal hysteresis may be due to the different load increase rate during step loading and constitutes the object of further study.

The relation between the observed BSC signal maxima that was mentioned before is valid for steps leading to small loads as well as for steps leading to large loads in the vicinity of the beam fracture. In general, the bending loading leads to a greater reliability of the macroscopic detection of cracks compared to the microscopic observed in PSC.

\section{Discussion - Conclusions}

The series of experiments has demonstrated there is clearly a mechanism that causes small electrical discharge currents generated by the motion of charged dislocations within loaded cementbased materials when strain changes occur, and that these discharge currents can be directly related to the magnitude of strains within the material. The theory of motion of charged dislocations (MCD) has been proposed, and this explains the phenomena that have been observed.

The experimental method requires great care, and at the moment is carried out in a laboratory, and inside a Faraday cage. The measurements are of very small currents - of the order of pico-amperes. Nevertheless, the signal is distinct, and the data presented here are not filtered in any sophisticated way. The noise/signal ratio may be of concern in more practical application, but as yet the 
portability of the technique and subsequent challenges has not been examined in detail. Thus basic signal data has been presented here and is adequate for assessment of initial relationships.

Irrespective of how promising the results of this technique might be for commercial applications, the authors acknowledge that significant development work needs to be undertaken before the technique can be applied in a wider commercial context.

In particular the ongoing preliminary work is now looking at the importance of reinforcement levels - with the obvious consequence of lower tensile strains - and the importance of positioning of sensors within the specimens. Further assessments that do not involve monotonic loading are essential. Statistical analysis that examines response to cumulative damage is beyond the scope of this paper.

Notwithstanding the challenges that remain, the possibilities for the application of the technique are exciting. The use of another in-situ non-destructive method for the measurement of strain levels in reinforced concrete structures is in itself worthwhile, but the potential to identify the reserves of strength in a concrete structures, as demonstrated by the change in characteristics at $70 \%$ and $90 \%$ of failure given in Figure 6 for PSC vs. stress, this would be an extremely useful goal for practical application in structural health monitoring.

The experimental results using the step stress technique indicate that electric current relaxation (fast or slow) after each abrupt stepwise stress increase depends significantly on the final mechanical loading level to which the sample is led. This is very interesting and after a detailed study, the determined value of relaxation time may constitute a measure of the damage within the material following the application of a step stress or even a stress pulse in the form of a delta function. Additional assessment methodologies without monotonic loading may prove to be of fundamental importance.

The results of the Bending Stimulated Current (BSC) technique are also interesting with the additional advantage of the simultaneous recording of two electrical signals emitted from both the tension and the compression zones of the sample.

Tests on specimens in uniaxial compression, and on specimens in three-point bending show repeatable characteristics. Analysis of these characteristics can lead to estimates of strain within the material, and the correlation of discharge currents with strain indicate possibilities for measuring reserve of strength.

While further work is undoubtedly needed - for example to examine reinforced concrete specimens - the benefits of transferring this nascent laboratory technique to an in-situ procedure for existing structures are evident. 


\section{References}

[1] Stepanov AW. Über den Mechanismus der plastischen Deformation. Z. Physics 1933; 81:560564.

[2] Whitworth RW. Charged dislocations in ionic crystals. Adv. Phys. 1975; 24:203-304.

[3] Finkelstein D, Hill RD, Powell JR. The piezoelectric theory of earthquake lightning. J. Geophys. 1973; 78:992-993.

[4] Nitsan U. Electromagnetic emission accompanying fracture of quartz-bearing rocks, Geophysical Research Letters 1977; 4:333-337.

[5] Enomoto J, Hashimoto H. Emission of charged particles from indentation fracture of rocks. Nature 1990; 346: 641-643.

[6] Varotsos P, Alexopoulos K.. Thermodynamics of point defects and their relation with bulk properties. North-Holland, Amsterdam; 1986.

[7] Slifkin L. Seismic electric signals from displacement of charged dislocations. Tectonophysics 1993; 224:149-152.

[8] Vallianatos F, Tzanis A. Electric current generation associated with the deformation rate of a solid: Preseismic and coseismic signals. Phys. Chem. Earth 1998; 23:933-938.

[9] Tzanis A, Vallianatos F. A physical model of electrical earthquake precursors due to crack propagation and the motion of charged edge dislocations, In: Hayakawa M, Molchanov OA, editors. Seismo Electromagnetics: Lithosphere-Atmosphere-Ionosphere Coupling, Terrapub, Tokyo; 2002, p.117-130.

[10] Vallianatos F, Tzanis A. A model for the generation of precursory electric and magnetic fields associated with the deformation rate of the earthquake focus, In: Hayakawa M, editor. Atmospheric and Ionospheric electromagnetic phenomena associated with Earthquakes, Terra Scientific Publishing Co, Tokyo; 1999, p.287-305.

[11] Vallianatos F, Triantis D, Tzanis A, Anastasiadis C, Stavrakas I. Electric Earthquake Precursors: From Laboratory Results to Field Observations. Physics and Chemistry of the Earth 2004; 29:339-351.

[12] Warwick JW, Stoker C, Meyer TR. Radio emission associated with rock fracture: Possible application to the great Chilean earthquake of May 22, 1960. J. Geophys. Res. 1982; 87:2851-2859.

[13] Ogawa TK, Miura T. Electromagnetic radiation from rocks. J. Geophys. Res. 1985;90:62456249.

[14] Enomoto J, Hashimoto H. Emission of charged particles from indentation fracture of rocks. Nature 1990; 346:641-643.

[15] Stavrakas I, Anastasiadis C, Triantis D, Vallianatos F. Piezo Stimulated currents in marble samples: Precursory and concurrent - with - failure signals. Natural Hazards and Earth System Sciences 2003; 3:243-247.

[16] Anastasiadis C, Triantis D, Stavrakas I, Vallianatos F. Pressure stimulated currents (PSC) in marble samples after the application of various stress modes before fracture. Annals of Geophysics 2004; 47:21-28.

[17] Stavrakas I, Triantis D, Agioutantis Z, Maurigiannakis S, Saltas V, Vallianatos F, Clarke M. Pressure stimulated currents in rocks and their correlation with mechanical properties. Natural Hazards and Earth System Sciences 2004; 4:563-567.

[18] Triantis D, Stavrakas I, Anastasiadis C, Kyriazopoulos A, Vallianatos F. An analysis of Pressure Stimulated Currents (PSC), in marble samples under mechanical stress. Physics and Chemistry of the Earth 2006;31:234-239.

[19] Triantis D, Anastasiadis C, Vallianatos F, Kyriazis P, Nover G. Electric signal emissions during repeated abrupt uniaxial compressional stress steps in amphibolite from KTB drilling. Natural Hazards and Earth System Sciences 2007; 7:149-154.

[20] Anastasiadis C, Triantis D, Hogarth CA. Comments on the phenomena underlying pressure stimulated currents (PSC) in dielectric rock materials. Journal of Materials Science 2007; 42:25382542. 
[21] Eccles D,_Sammonds PR, Clint OC. Laboratory studies of electrical potential during rock failure. International Journal of Rock Mechanics \& Mining Sciences 2005;42 :933-949.

[22] Aydin A, Prance RJ, Prance H, Harland CJ. Observation of pressure stimulated voltages in rocks using an electric potential sensor. Applied Physics Letters 2009;95(12), art. no. 124102.

[23] Vallianatos F, Triantis D. Scaling in Pressure Stimulated Currents related with Rock Fracture. Physica A 2008; 387:4940-4946.

[24] Wittmann FH. Observation of an electromechanical effect of hardened cement paste. Cem. Concr. Res. 1973; 3:601-605.

[25] Kyriazopoulos A, Anastasiadis C, Triantis D, Stavrakas I. Experimental Pressure Stimulated Currents (PSC) recordings on cement mortar under mechanical stress. Proceedings of the 2nd International FIB Congress, Naples, Italy 2006:ID 13-19.

[26] Kyriazopoulos A, Stavrakas I, Anastasiadis C, Triantis D., Pressure stimulated current emissions on cement paste samples under repetitive stepwise compressional loadings. Journal of Mechanical Engineering, 2010; 56 (9): 551-556.

[27] Sun M, Liu Q, Li Z, Wang E. Electrical emission in mortar under low compressive loading. Cement and Concrete Research 2002;32: 47-50.

[28] Sun M, Li Z, Song X. Piezoelectric effect of hardened cement paste. Cement \& Concrete Composites 2004;26:717-720.

[29] Sun M, Liu Q, Li Z, Hu Y. A study of piezoelectric properties of carbon fiber reinforced concrete and plain cement paste during dynamic loading. Cement and Concrete Research 2000;30:1593-1595.

[30] Triantis D, Anastasiadis C, Stavrakas I, Kyriazopoulos A. The Ascertainment of the Presence of Damage Processes Using the Pressure Stimulated Current (PSC) Technique on Marble and Cement Samples. Proceedings of the 9th ECNDT, Berlin 2006;Tu.1.6.2.

[31] Kyriazopoulos A. Mechanical stress induced electrical emissions in cement based materials. PhD Thesis:Brunel University, 2009

[32] Kyriazopoulos A, Stavrakas I, Anastasiadis C, Triantis D. The correlation of the electric current peaks emitted from stressed hardened cement paste with the strain rate. Proceedings of the Concrete: $21^{\text {st }}$ Century Superhero, 22-24 June, London UK. 2009.

[33] Triantis D, Anastasiadis C, Stavrakas I. The Correlation of Electrical Charge with Strain on Stressed Rock Samples. Natural Hazards and Earth System Sciences 2008; 8:1243-1248.

[34] Turcotte D. L. and Shcherbakov R. Can damage mechanics explain temporal scaling laws, in: brittle fracture and seismicity?. Pure Appl. Phys. 2006; 163:1031-1045.

[35] Design and Control of Concrete Mixtures, $14^{\text {th }}$ edition, Portland Cement Association. 14th ed. (February 2002). By S.H. Kosmatka, B. Kerkhoff, and W.C. Panarese.

[36] Griffith A.A. The phenomena of rupture and flow in solids. Philosophical Transactions, The Royal Society of London Series 1920; A221:163.

[37] Saito M. Tensile fatigue strength of the mortar-aggregate bond. Cement and Concrete Research, 1988; 18:710-714.

[38] Landis E, Baillon L. Experiments to Relate Acoustic Emission Energy to Fracture Energy of Concrete. Journal of Engineering Mechanics 2002; 128:698-702. 


\section{Figure Captions}

Fig. 1. Experimental arrangement (not to scale) to perform PSC experiments

Fig. 2. Experimental arrangement (not to scale) to perform BSC experiments

Fig. 3. Stress and strain with respect to time

Fig. 4. The stress - strain diagram of the sample used in the experiment

Fig. 5. PSC behaviour related to constantly increasing stress and to the corresponding strain. It is evident that when the strain curve departs from linearity, PSC is emitted

Fig. 6. Variation of PSC on a logarithmic scale after noise elimination with respect to the relative compressive stress

Fig. 7. Two PSC recordings at different stress rates with respect to time on identical samples

Fig. 8. PSC recordings due to four consecutive abrupt stress steps on cement mortar samples

Fig. 9. PSC peaks: after abrupt stress increase (peak 1) and PSC peak after continuous constant stress (peak 2). The third and final peak after stress step 2 signals failure

Fig. 10. PSC and strain variations depicted with respect to time

Fig.11. PSC due to four successive stress steps depicted with respect to time

Fig. 12. PSC and strain variations due to four successive stress steps depicted with respect to time

Fig. 13. Behaviour of the PSC peak values of two different experiments on identical samples shows that PSC peaks constitute a linear function of strain rate

Fig. 14. BSC and loading force on a beam, depicted with respect to time

Fig. 15. BSC and loading force on a beam applied in three consecutive steps, depicted with respect to time 
Table 1

\begin{tabular}{|c|c|c|}
\hline Class & Dimensions (mm) & Fracture limit \\
\hline CM60 $^{[1]}$ & $50 \times 50 \times 60$ & $35-41 \mathrm{MPa}$ \\
\hline $\mathrm{CM}^{\mathrm{CII}}$ & $50 \times 50 \times 50$ & $25-30 \mathrm{MPa}$ \\
\hline CM70 ${ }^{[1]}$ & $50 \times 50 \times 70$ & around $55 \mathrm{MPa}$ \\
\hline CM160 ${ }^{[2]}$ & $40 \times 40 \times 160$ & $(1.4-1.5 \mathrm{kN})$ \\
\hline
\end{tabular}

[1] PSC experiments

[2] Three point bending (BSC) experiments 
Figure 1

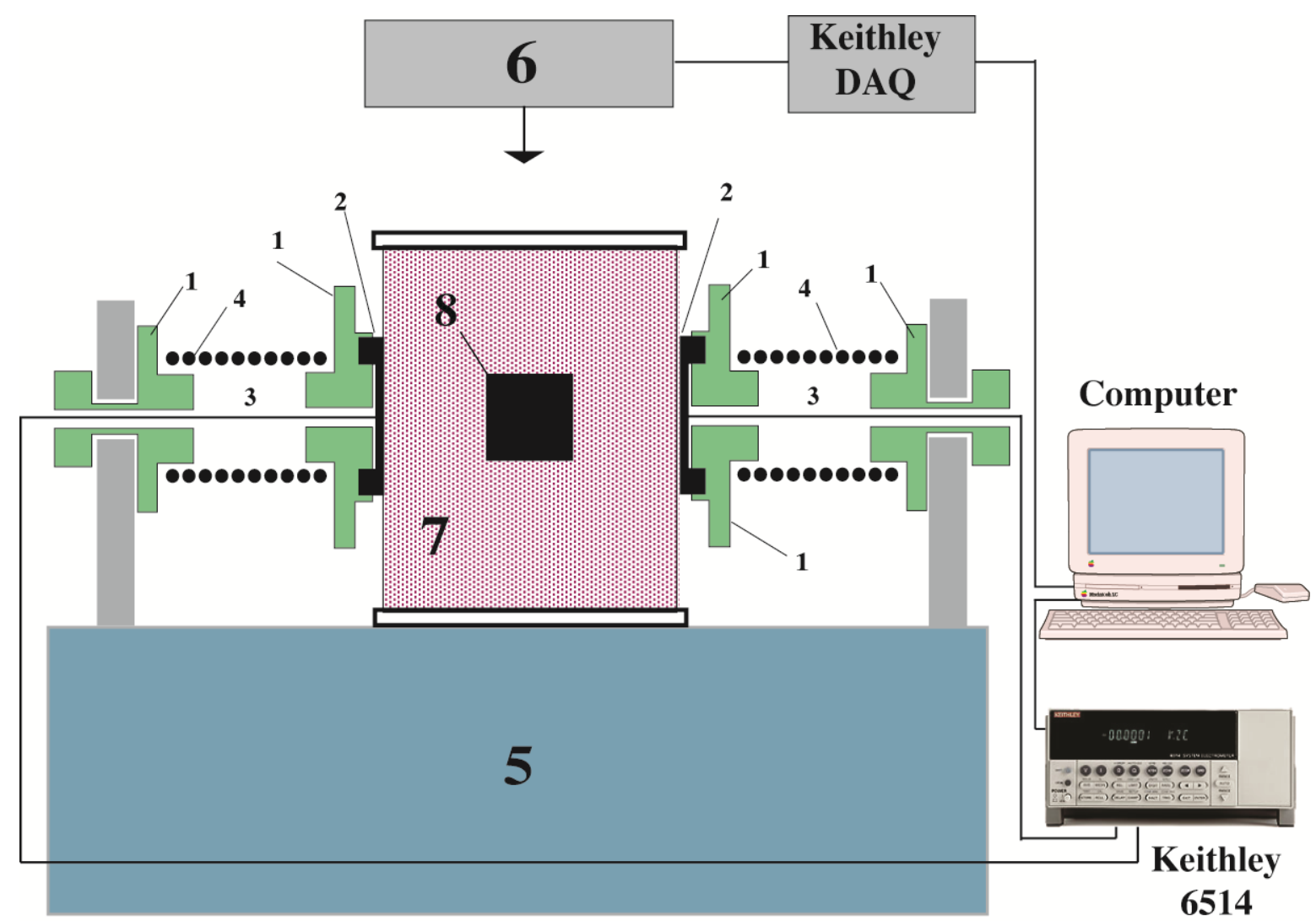

1. Teflon (electric insulator)

5. Steel base

2. Copper electrodes

6. Load cell

3. Leads to the electrometer

7. Sample

4. Spring holder

8. Strain gauge

6514 
Figure 2

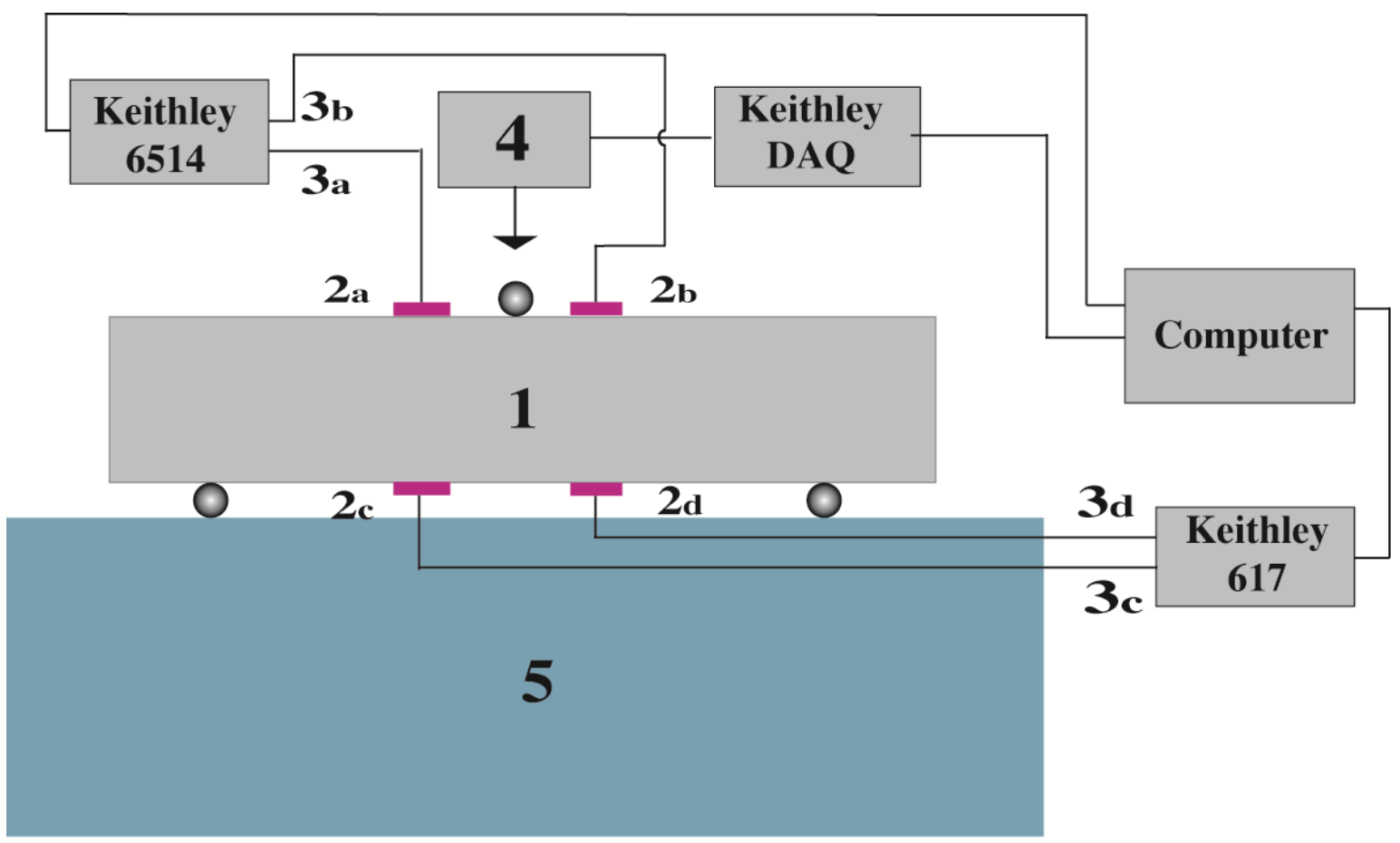

1. Sample

4. Load cell

2 (a,b,c,d) Copper electrodes

5. Steel base

3 (a,b,c,d) Leads to the electrometers 
Figure 3

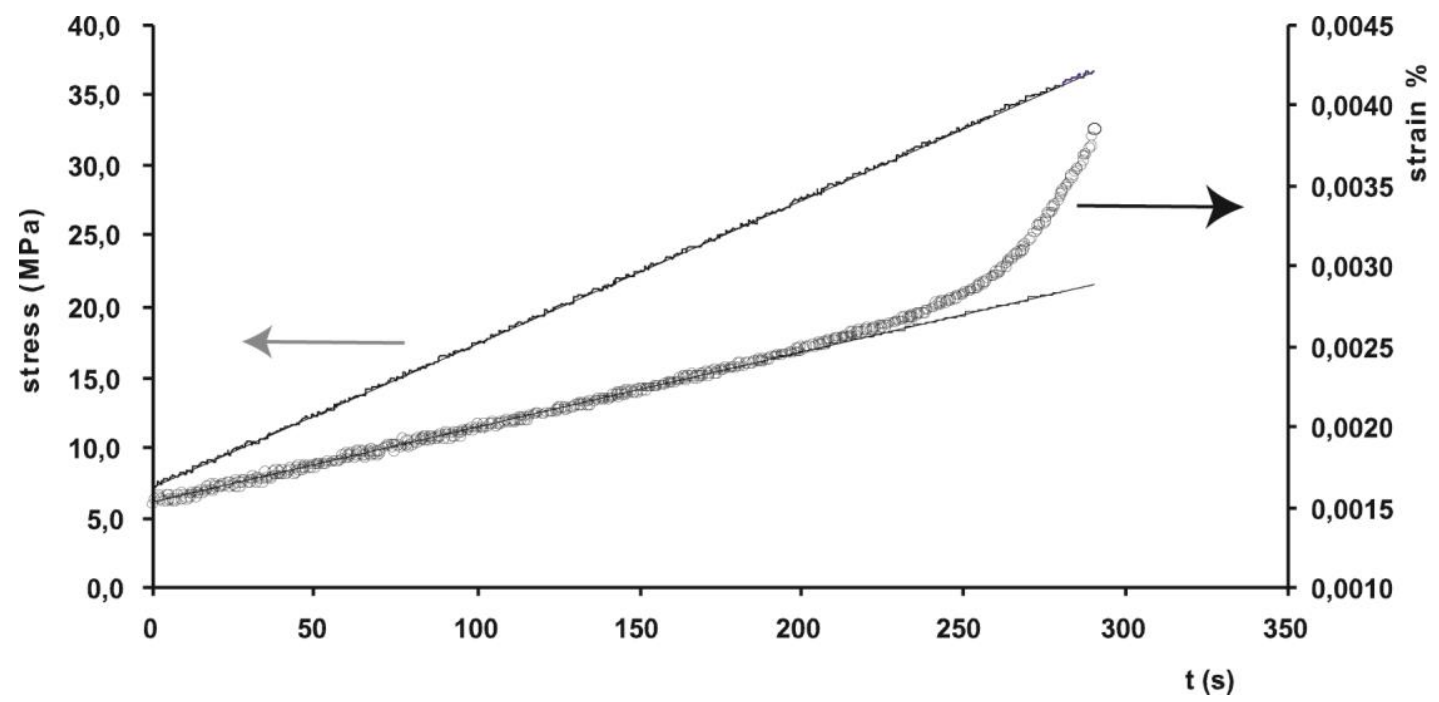

Figure 4

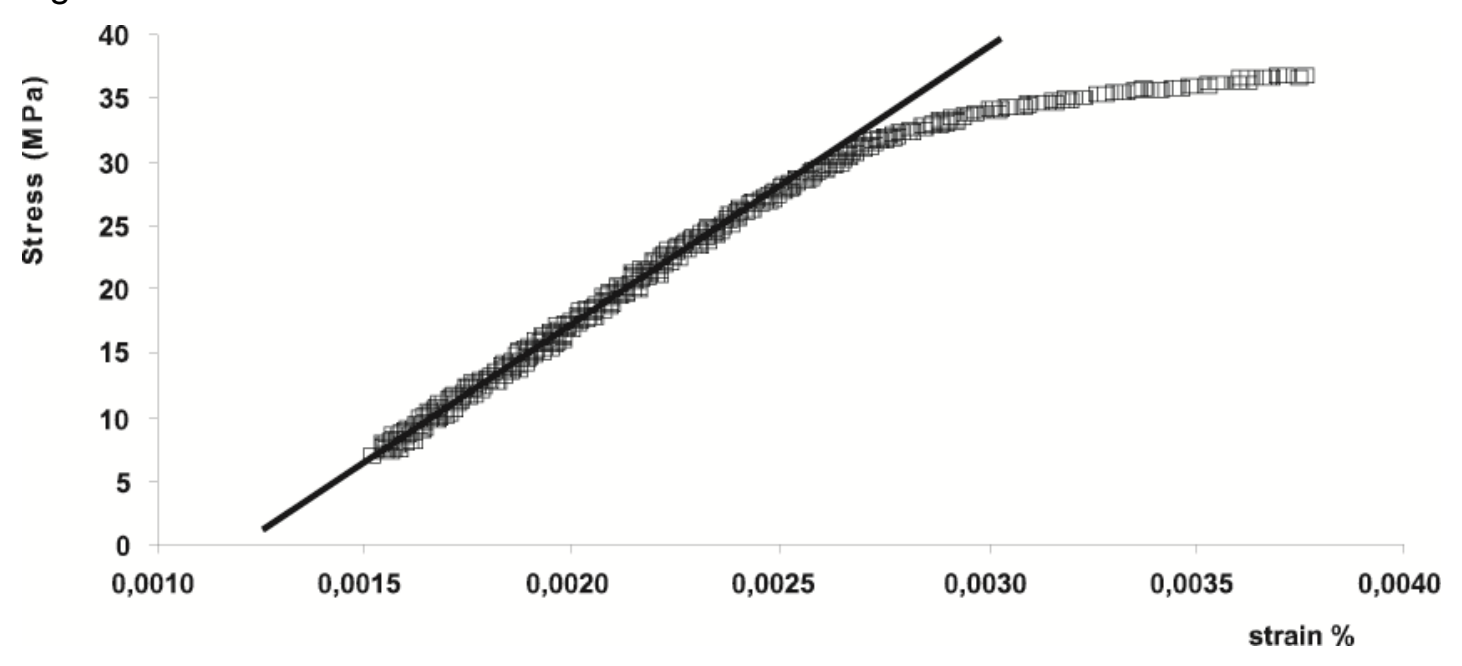


Figure 5

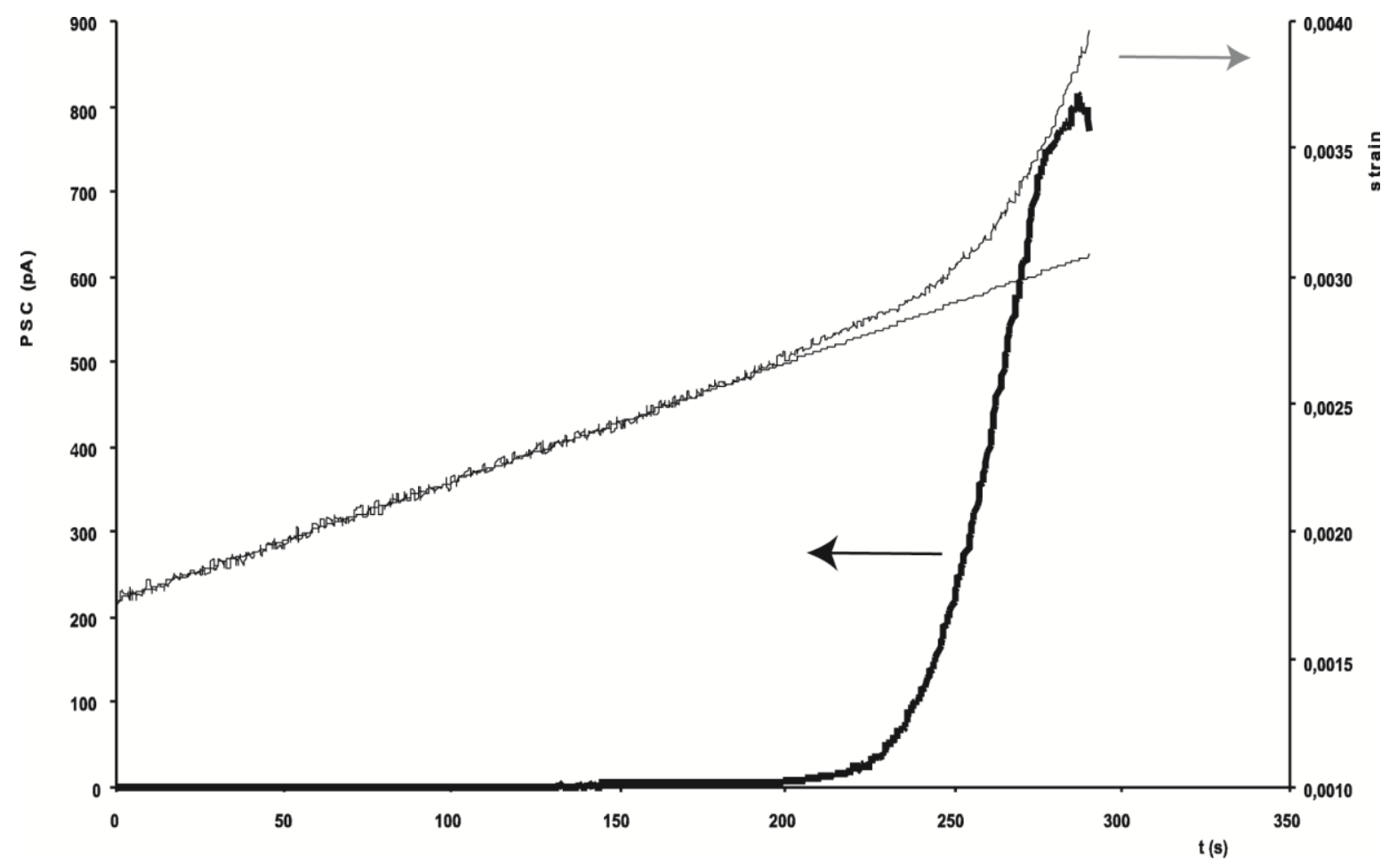

Figure 6

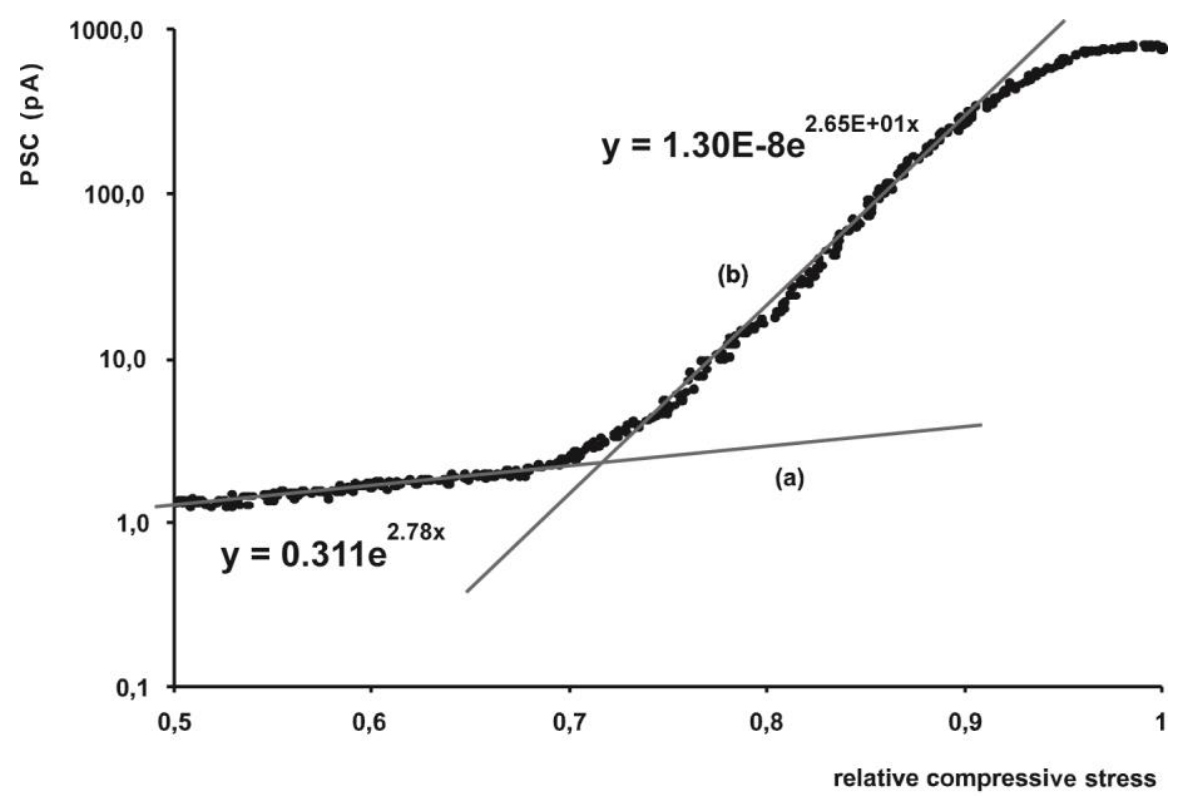


Figure 7

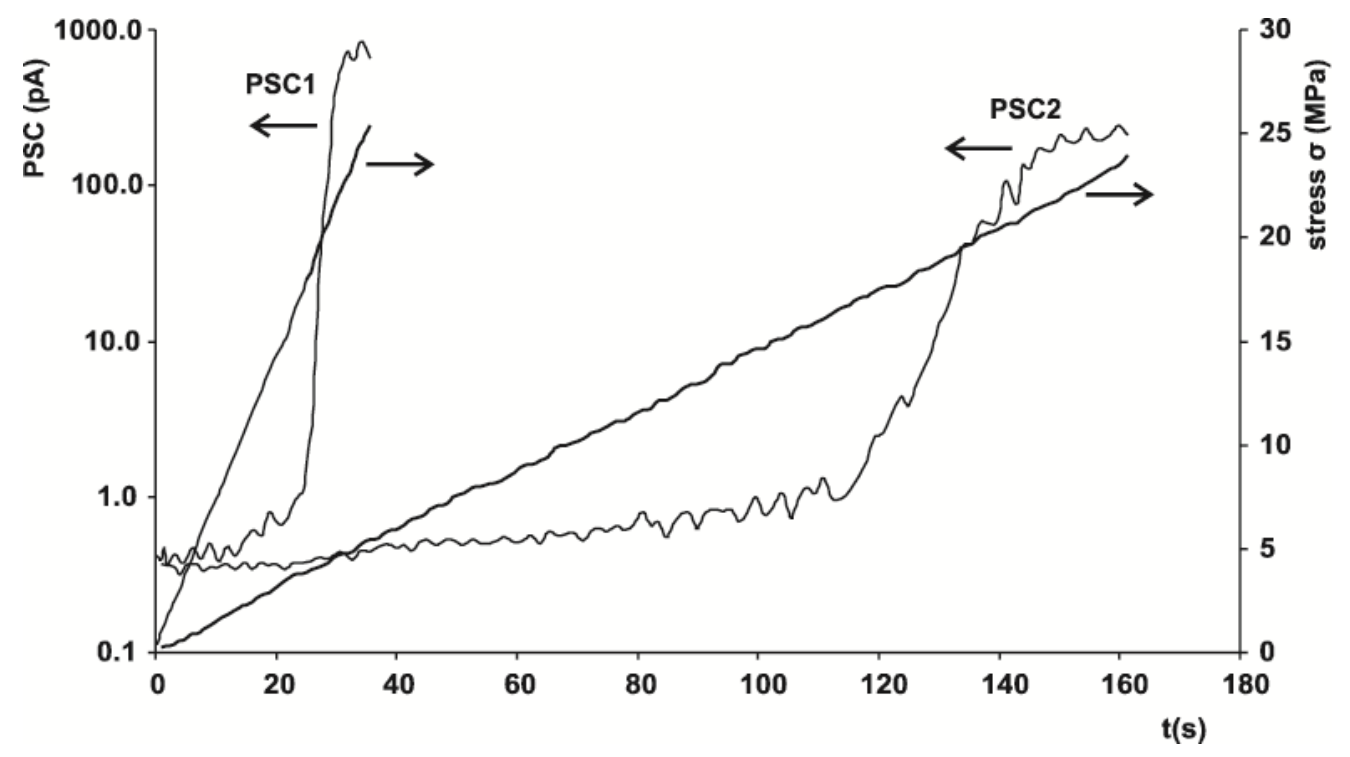

Figure 8
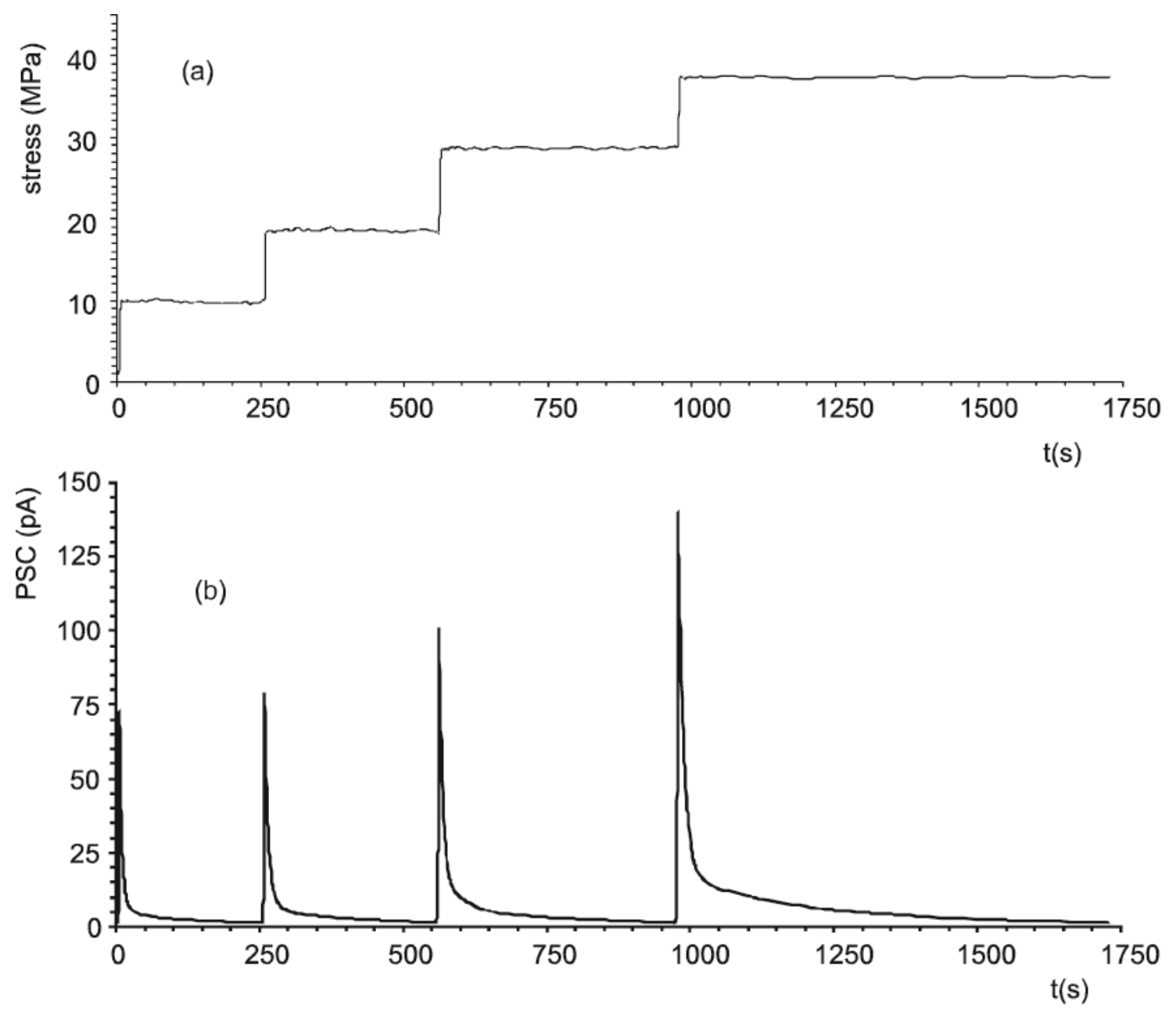
Figure 9

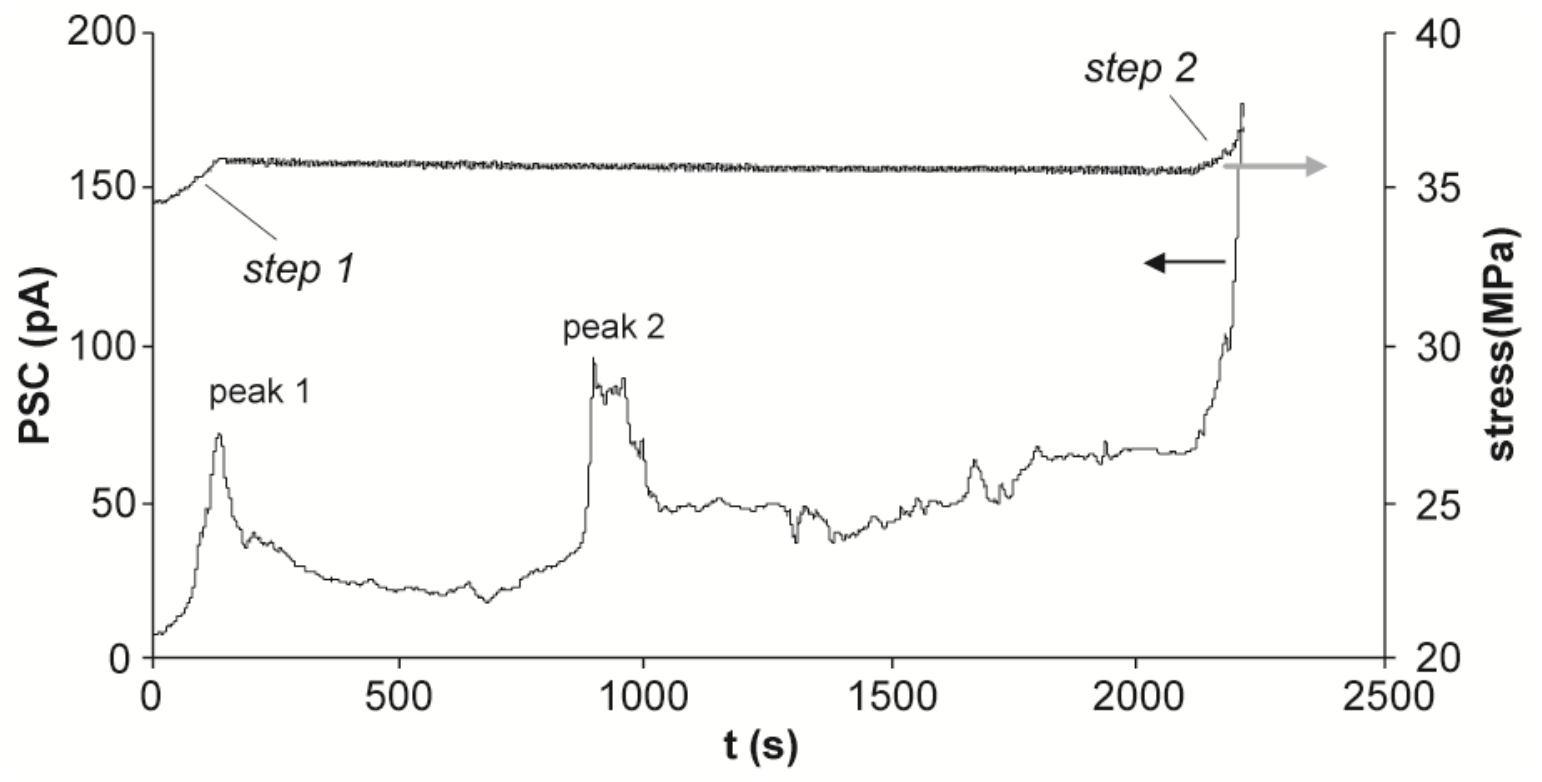

Figure 10

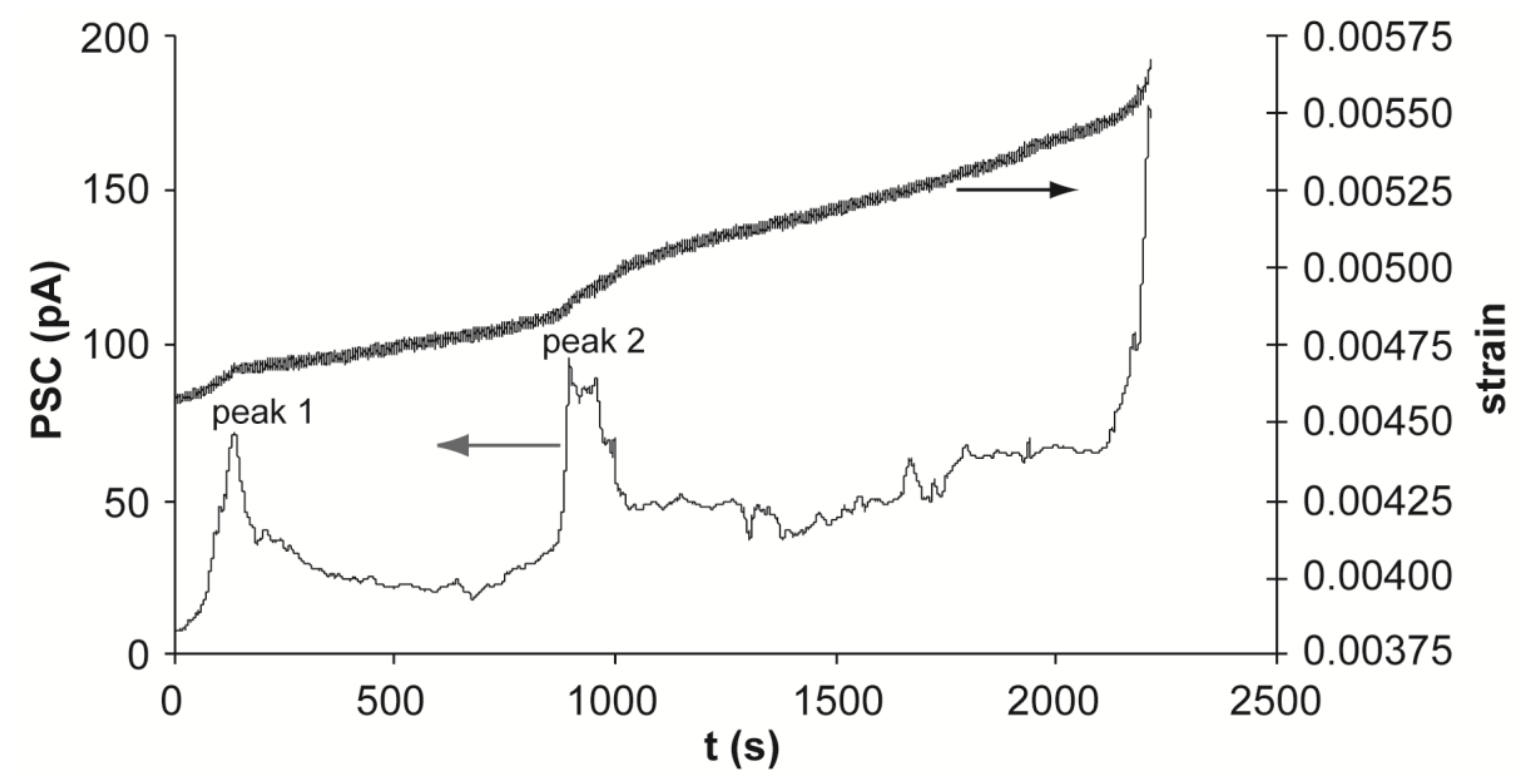


Figure 11

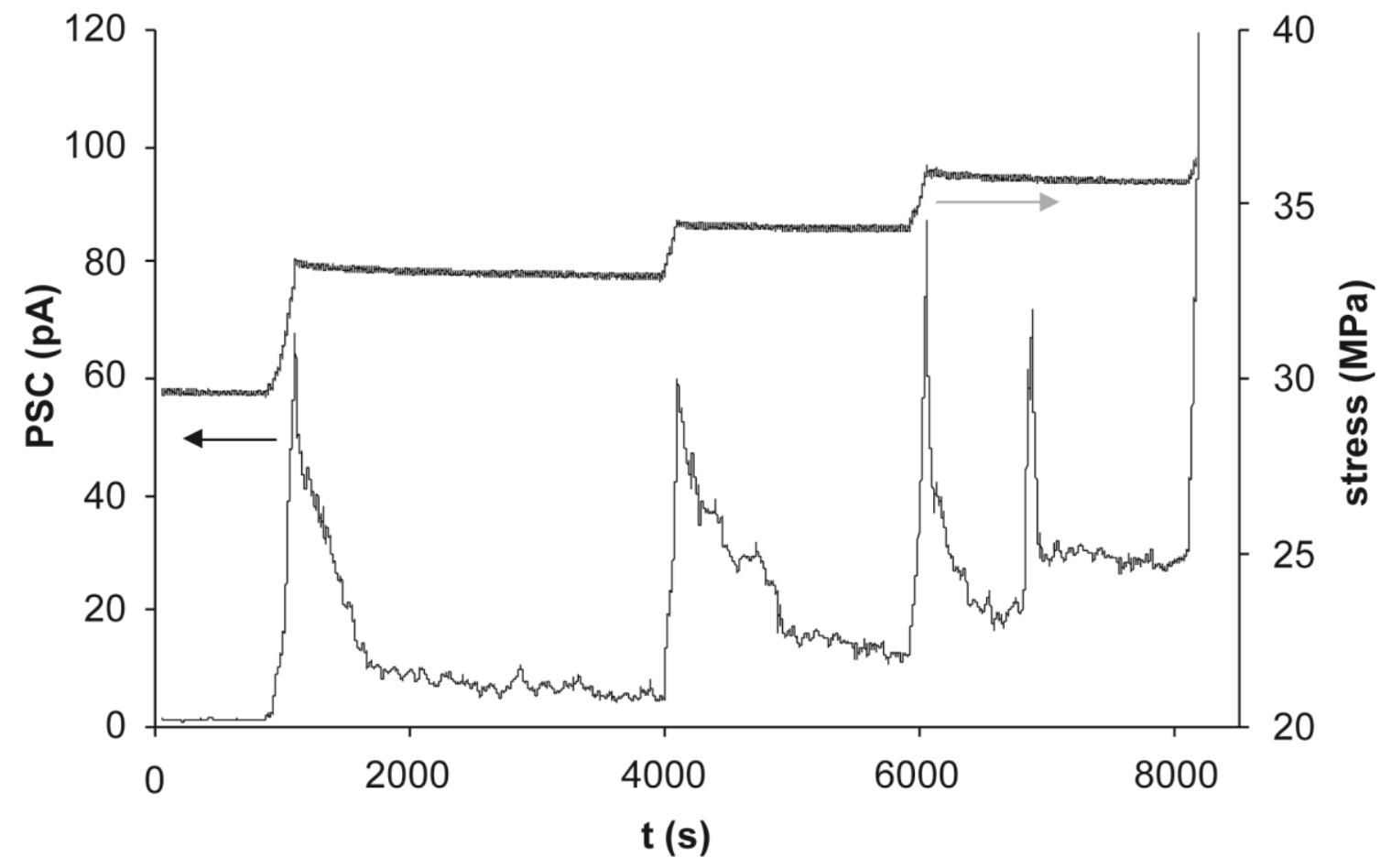


Figure 12

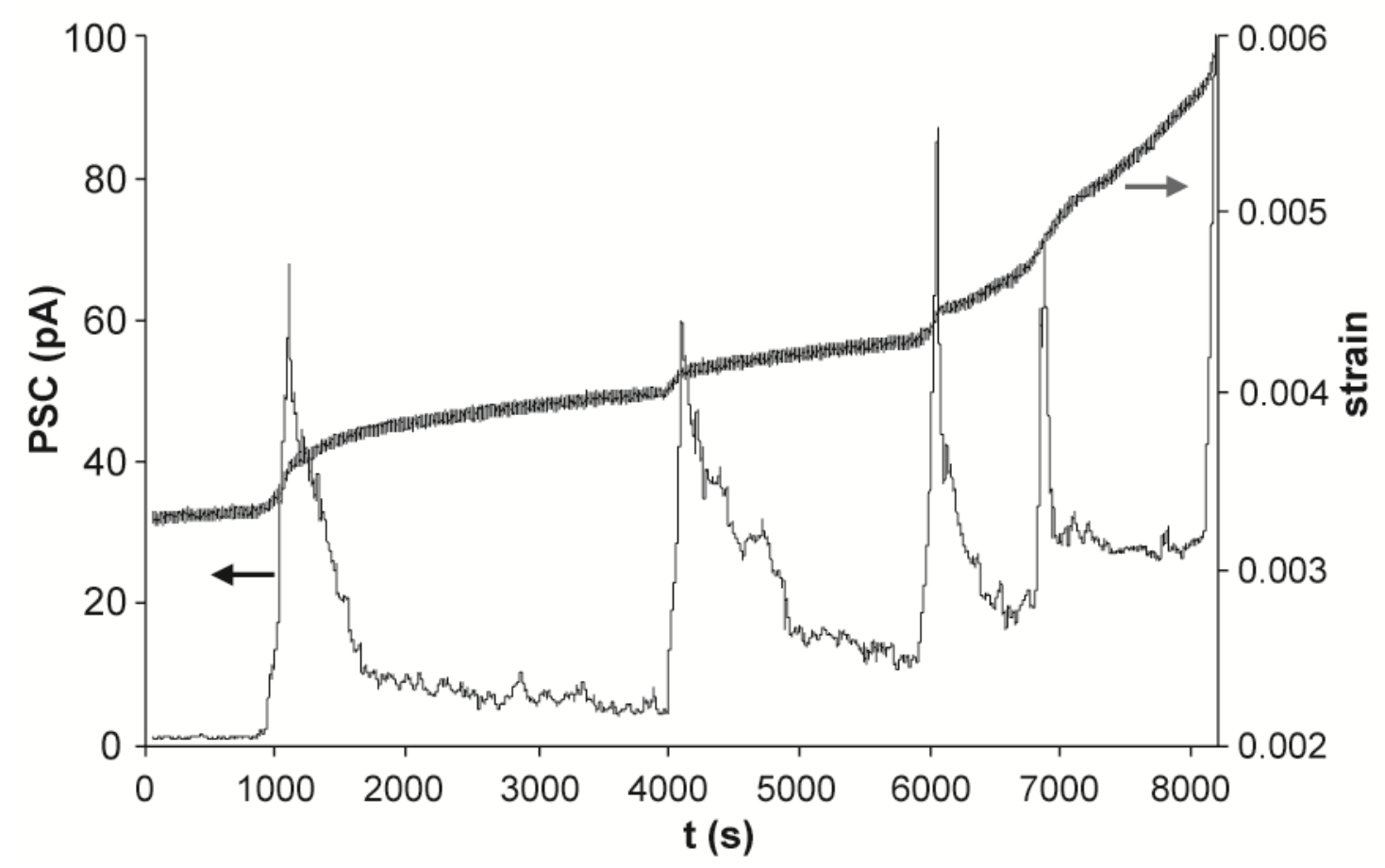

Figure 13

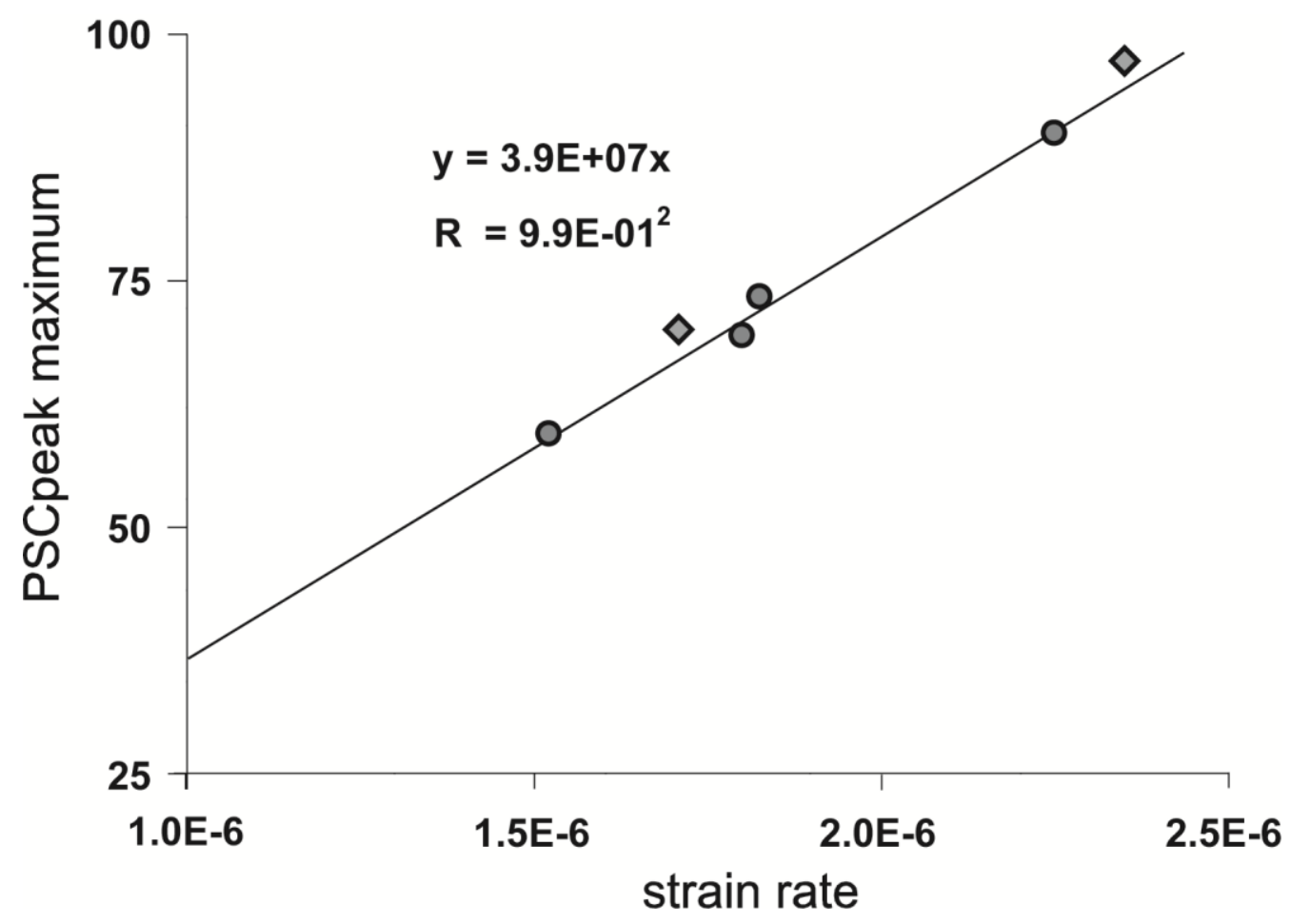


Figure 14

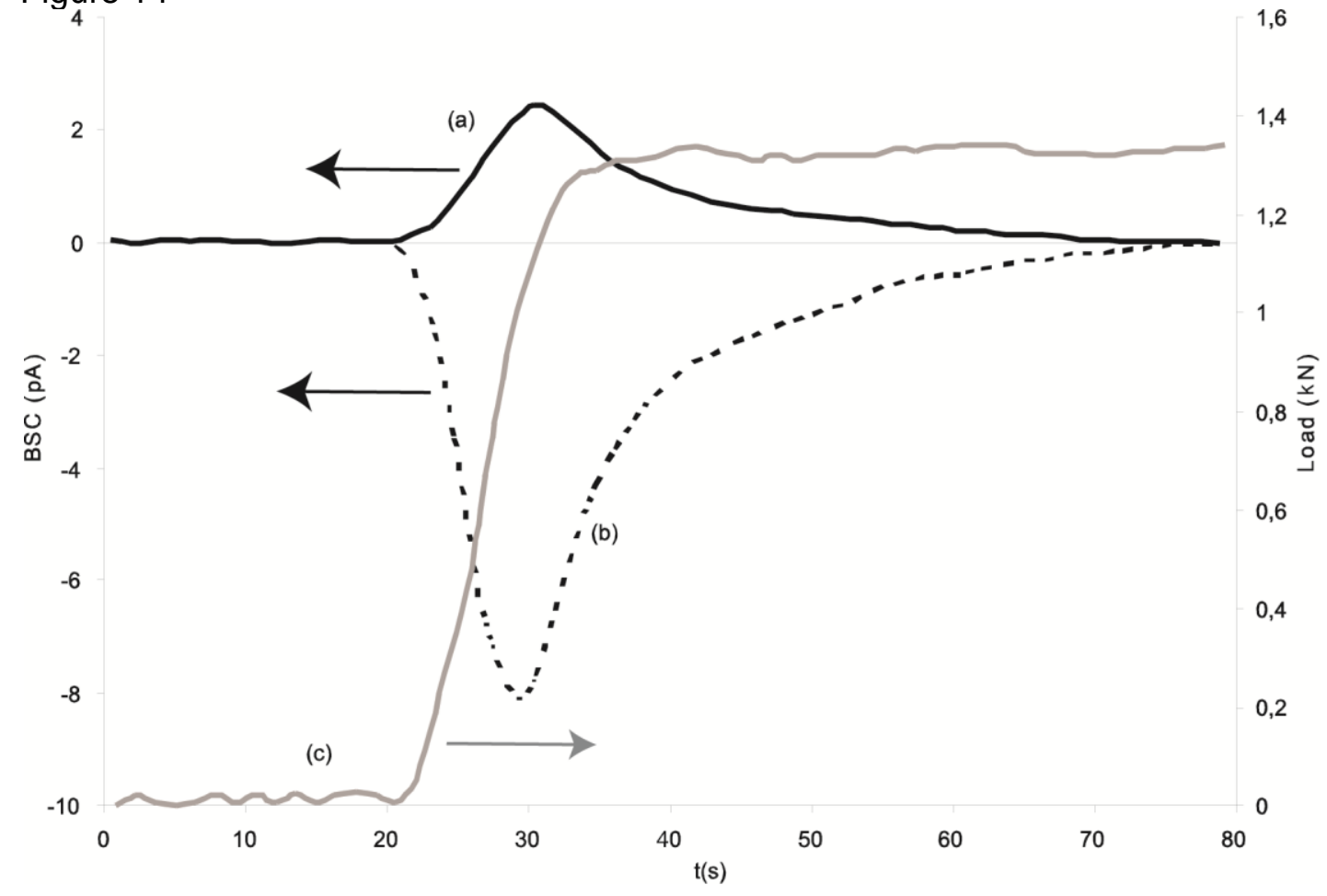

Figure 15

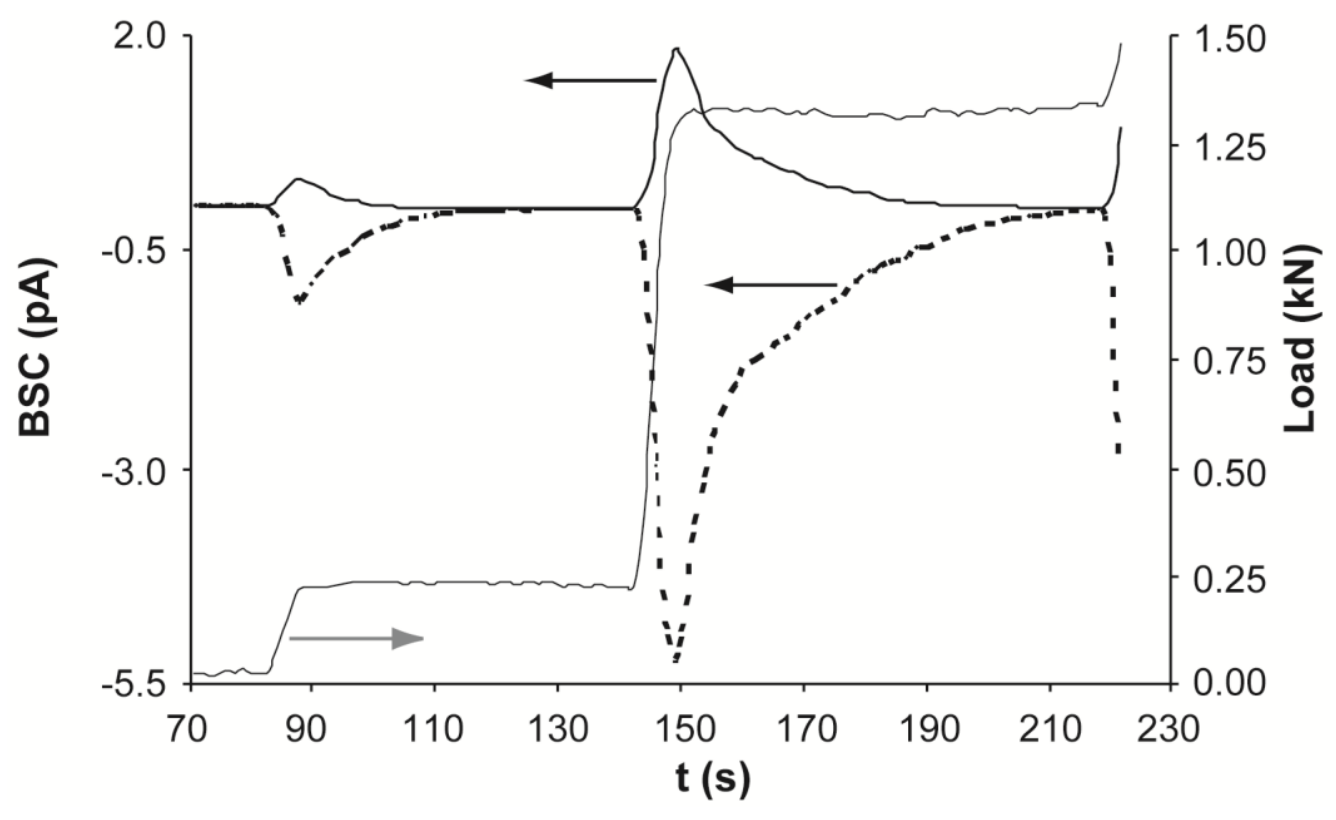

